\title{
Reflexión sobre el clima organizacional, la percepción del colaborador y la gestión del talento humano
}

Reflection on the organizational climate, the perception of the collaborator and the management of human talent

Flor Alba Pinzón Acosta

Administradora de Empresas, especialista en Docencia Universitaria, Mg. en Educación y Mg. en Dirección Estratégica, Gerencia del Talento Humano y Gestión del Conocimiento. Docencia en postgrado, pregrado e investigación. En modalidad presencial y a distancia, Gestión académica en Educación Superior Procesos de Registros Calificados programas ante el MEN y Acreditación de Alta Calidad. Procesos de articulación educación media y superior. Diseño curricular para programas de pregrado en modalidad presencial y virtual. Diseño de programas de Administración de Empresas por ciclos propedéuticos y basados en competencias.

Correo electrónico: floralbapinzon@ustadistancia.edu.co 


\section{Resumen}

Alcanzar excelentes indicadores en la gestión de una organización, supone un interés sin límites por afinar la dirección que se ejerce en el componente humano, la disposición de los equipos y grupos de trabajo, y su interdependencia con las individualidades de cada persona, facilitando la identidad y balance entre los objetivos, intereses personales y colectivos, junto con los objetivos e intereses organizacionales.

De esta manera, el clima organizacional representa una inversión a largo plazo para las organizaciones, dado que el talento humano es la base competitiva de las empresas, junto con el desarrollo de los sistemas de información, el alto nivel de integración y el proceso de globalización que se ha observado en las últimas décadas. Por lo tanto, la necesidad de medir el impacto del clima organizacional para entender los elementos, características y variables propias de las organizaciones, es un tema que sigue despertando el interés profesional y de diversas disciplinas, como las ciencias políticas, la antropología, la psicología, entre otras.

Teniendo en cuenta lo anterior, se hace necesario que para llevar a cabo el análisis de las diversas problemáticas sobre las dinámicas organizacionales dadas por los cambios sociales, económicos, políticos y culturales, que generan impacto en el clima organizacional, se deban establecer procesos que conlleven a la aceptación y apropiación de los colaboradores de la organización, que se argumenten teórica y empíricamente con una metodología y estructura propia al contexto de la organización, que permita abordar y explicar los fenómenos que se originan a partir de cada una de las actividades organizacionales, tales como el cambio, la adaptación y el aprendizaje, destacables en entornos flexibles.

Palabras clave: organización, clima organizacional, medición, talento humano, colaborador.

\section{Abstract}

Achieving excellent indicators in the management of an organization, is an unlimited interest in fine-tuning the direction in the human component, the provision of teams and working groups, and their interdependence with the individualities of each person; Facilitate the identity and balance between objectives, personal and collective interests, along with organizational objectives and interests.

In this way, the organizational climate represents a long-term investment for organizations, given that human talent is the competitive base of companies, along with the development of information systems, the high level of integration and the globalization process Which has been observed in the last decades. Therefore, the need to measure the impact of organizational climate to understand the elements, characteristics and variables of organizations is an issue that continues to arouse professional interest and diverse disciplines, such as political science, anthropology, psychology, among other.

It is necessary that in order to carry out the analysis of the various problems on the organizational dynamics given by the social, 
economic, political and cultural changes that have an impact on the organizational climate, it is necessary to establish processes that lead to the acceptance and appropriation of the Collaborators of the organization, who argue theoretically and empirically with a methodology and structure proper to the context of the organization that allows to approach and explain the phenomena that arise from each of the organizational activities, such as change, adaptation and Learning in flexible environments.

Keywords: organisation, organisational climate, measurement, human talent, collaborator.

\section{Introducción}

Lograr un clima organizacional óptimo es una inversión a largo plazo para las organizaciones, ya que el talento humano es la base competitiva de las empresas, por lo tanto, en los tiempos actuales, es de gran importancia valorarlo con una adecuada atención ya que esto permite identificar la percepción que tienen los colaboradores de la organización donde se trabaja y a su vez apreciar la gestión del talento humano que hacen los directivos frente a su capital intangible más valioso, es decir, las personas. Este primer acercamiento tiene como finalidad entender y dar a conocer cómo la percepción que forma el colaborador o empleado miembro de cualquier organización impacta el clima organizacional. Esto se configura desde la gestión de los directivos o miembros responsables de la estructuración de la empresa, a través de las evidencias de la medición de algunos indicadores, conformados para este fin.
El clima organizacional es una dimensión muy vulnerable que, junto con el desarrollo de los sistemas de información, el alto nivel de integración y el proceso de globalización, que se ha observado en las últimas décadas, además de los profundos y rápidos cambios en el escenario político y económico, el fracaso relativo de las economías europeas y norteamericana y el avance de las economías asiáticas (Japón, Taiwán, Corea, etc.), son factores, que han llevado a que las decisiones sobre los cursos de acción por tomar e inducir en las organizaciones se hayan vuelto más complejas, dinámicas y desafiantes; lo cual hace necesario tener control sobre una gran cantidad de aspectos, que no son de poca importancia. En este contexto, el fenómeno cultural ofrece un punto de apoyo de relativa estabilidad, que permite re-pensar la organización y sus modos de vinculación con el entorno (Naranjo, 2014).

Este planteamiento demanda el desarrollo organizacional, conformado como mínimo por tres elementos que permiten la duración o existencia de cualquier organización: primero, los requerimientos para subsistir en condiciones satisfactorias; segundo, las exigencias del medio en el que la organización se desenvuelve, es decir, leyes y reglamentos, exigencias del mercado, opinión pública, entre otras; y tercero, los requerimientos individuales y sociales del recurso humano que conforma los equipos y permite el desarrollo de la organización que se evidencian en el clima organizacional que la conforma.

Según Mladinic (2011), el clima organizacional refleja el impacto que generan los resultados de múltiples acciones y prácticas organizacionales que son percibidas por los trabajadores como "justas y equitativas", ta- 
les como la asignación de cargas de trabajo, el pago justo de salario y prestaciones, promociones equitativas, trato digno y justo para todos los colaboradores, entre otras. En este sentido, la percepción de justicia organizacional se verá reflejada desde los resultados y desempeño del colaborador, lo cual incide directamente en la organización, en los índices de rotación, el desempeño, la satisfacción laboral, la productividad, entre otros (Patlán-Pérez, Martínez \& Hernández, 2012).

Como bien lo dicen Patlán-Pérez, Martínez \& Hernández (2012), la “justicia organizacional se plantea como un antecedente de variables tales como compromiso organizacional, satisfacción laboral y productividad" (p.4).

La inequidad o injusticia organizacional se evidencia en la falta de productividad, disminución de la calidad en la producción y prestación de servicios y en el incremento de los índices de ausentismo. Dado que la justicia social es percibida por el colaborador, dependiendo de su concepto frente a lo justo y lo injusto, en la relación obrero-patrón que se desarrolle en la organización, si el colaborador percibe que es justamente tratado, tendrá actitudes positivas ante el trabajo, el jefe y los demás miembros de la organización; en una situación opuesta, si la percepción es negativa generara tensiones, sentimientos de insatisfacción y desmotivación por parte del colaborador de la organización.

Cabrera (1999), dice que el clima organizacional es uno de los aspectos más considerados en las concepciones sobre los sistemas de desarrollo humano. Quizás, como ningún otro parámetro, desde allí se define la esencia para el desarrollo de las organizaciones. Además de expresar los indicadores de productividad, eficiencia, competitividad, cultura, desempeño, satisfacción laboral entre otros, que deben ponerse de manifiesto en una empresa o institución para lograr ubicar a hombres y mujeres, en todas sus dimensiones, en la cima laboral de la organización.

Desde las dinámicas de investigación iniciales que vinculan el clima, la percepción del empleado y las buenas prácticas directivas, se proponen algunos interrogantes que se resolverán en una segunda fase que tiene como objetivo crear un modelo que permita generar escenarios apropiados para un buen uso del clima organizacional: ¿Qué estructura y componentes debe tener un modelo para determinar el clima organizacional? ¿Cuál debe ser el papel de éste dentro de la creación de nuevas formas de concebir la organización para un mejor desarrollo?

Estos cuestionamientos surgen desde la necesidad de crear un modelo que permita entender las dimensiones del clima organizacional dentro de la dinámica y las buenas prácticas empresariales. Se podría, entonces, entender que el clima organizacional se observa como una descripción neta y global de la relación que existe entre colaborador y organización, haciendo referencia a la manera como ésta se puede percibir, teniendo como base su posición dentro de la misma; a partir de allí, se busca dar inicio a la construcción de un modelo que permita identificar las estructuras y los parámetros que rigen las instituciones, con el fin de influir en la satisfacción, tanto laboral como personal, de los empleados. Uno de los enfoques por desarrollar, es la forma en la que se pueden medir las respuestas afectivas que se generan entre los 
individuos, una vez el entorno sea asimilado por los mismos.

Teniendo en cuenta que la satisfacción es la medida o expresión más importante en la construcción de un buen clima organizacional, merece la pena evaluar el grado de satisfacción laboral para valorar las organizaciones y lo referente al ambiente laboral en el que se encuentra inmersa la institución, que depende de la capacidad que se tenga para dirigir de manera participativa la empresa.

El clima organizacional es el reflejo del ambiente organizacional que perciben o experimentan los miembros de la organización y que influye en su comportamiento. Este es favorable cuando la empresa proporciona satisfacción a las necesidades personales y a la elevación moral de los miembros, y desfavorable cuando no se logra satisfacer dichas necesidades (Chiavenato, 2002)

Para que una organización logre la productividad y sostenibilidad, es necesario que se proporcione una continuidad en los procesos, teniendo como prioridad la actuación, es decir, la atención, el conocimiento, la efectividad en la respuesta de cara a los clientes, -que debe originarse cada vez más-, de los empleados que están cerca de los procesos internos y de los mismos clientes de la organización. Este cambio se logra enriqueciendo el factor humano de la organización a partir de capacitaciones, desarrollo de habilidades y competencias para que su mente y capacidades puedan ser movilizadas hacia el logro de los objetivos empresariales. Los esfuerzos para mejorar la vida laboral deben ser sistemáticos y así lograr frente a los empleados una verdadera oportunidad de mejora que genere mayor confianza y compromiso ante su puesto de trabajo y su contribución a la empresa.

El interés de las empresas que buscan mejorar los niveles de eficiencia y productividad mediante la percepción y sentido de pertenencia de los colaboradores de las mismas, se ha incrementado potencialmente. Se considera entonces que el clima organizacional permite describir y diferenciar una empresa frente a otra, buscando crear un impacto en el comportamiento de sus integrantes, congregando aspectos como estructura, política, estilo de dirección, liderazgo, conflictos, prácticas, métodos de recompensas, castigos, control y supervisión de la organización. Igualmente, el clima organizacional impacta en la cultura, los valores institucionales, el sentido de pertenencia y la satisfacción laboral.

En este contexto, y teniendo presente que se han realizado un sin número de investigaciones respecto a ello, se desarrolla el proyecto "Diseño modelo para medición y determinación del clima organizacional" para ser aplicado en diferentes contextos empresariales, partiendo de que uno de los objetivos clave de esta iniciativa es detectar las dimensiones, categorías y variables que pueden impactar en el comportamiento requerido por los integrantes de las organizaciones para lograr las metas establecidas que le permitan alcanzar los estándares requeridos para ser organizaciones competitivas. Con estas pautas, se busca construir una propuesta que permita identificar una realidad empresarial, es decir, el clima organizacional, que sea reconocida por las organizaciones que estén dispuestas para establecer planes de mejoramiento necesarios para lograr el fortalecimiento requerido en el talento humano, lo cual permite aumentar la 
productividad y llevar a la empresa a consolidarse dentro del mercado como una organización competitiva.

Para lograr este objetivo, se busca diseñar y validar un modelo para medición del clima organizacional en diferentes contextos empresariales, estableciendo las dimensiones y variables que impactan en la percepción de los colaboradores, apoyándose en una revisión teórica para la elaboración de un instrumento como herramienta de medición, con el fin de confirmar la premisa que las dimensiones propuestas en el modelo, deben lograr identificar elementos subjetivos y objetivos que dan origen al clima organizacional de las diferentes empresas, y que quienes apliquen la propuesta, obtengan de ésta herramientas, estrategias de mejoramiento y fortalecimiento en los niveles de productividad, efectividad y competitividad.

La primera fase de la investigación se desarrolla en la respuesta a ciertos interrogantes como ¿para qué, por qué y cómo medir el clima organizacional en las empresas y cuál es el impacto que tiene la gestión del talento humano en el clima organizacional?

\section{Para qué medir el clima organizacional y la importancia de su medición}

En esta primera parte se hará una introducción desde diferentes aportes teóricos teniendo como base la definición de clima organizacional y la necesidad de medir su impacto para entender cuáles son los elementos, características y variables establecidas para este tipo de estudios. El clima organizacional según Robbins (2004), es un tema que sigue despertando el interés profesional y de diversas disciplinas, como la ciencia política, la antropología, la psicología, la psicología social, la sociología, la sociología organizacional, entre otras, bajo una premisa común que es interpretar y reconocer la importancia del fenómeno en el que priman las interacciones trabajador-organización y trabajador-trabajador. Esencialmente, la incidencia que tiene la organización en aspectos de carácter psicológico dentro de los individuos y sus interacciones permite la formulación del concepto de clima organizacional y se relaciona con factores como cultura, comunicación y motivación, entre otros.

Weinert (citado por Seisdedos, 1985) señala tres distintas escuelas en la concepción del clima organizacional:

La primera es concebida como un conjunto de características objetivas de la organización, perdurables y fácilmente medibles, que distinguen una entidad laboral de otra. Estas son los estilos de dirección, las normas y el medio ambiente fisiológico, las finalidades y los procesos de contraprestación. Aunque en su medida interviene la percepción individual, lo fundamental son los índices objetivos de dichas características. En esta línea están los trabajos de Forehand \& Gilmer (1965), de Litwin (1966) y Stringer (1968) (Seisdedos, 1985).

La segunda escuela se entiende desde una serie de variables perceptivas o atributos cualitativos de la organización. Estos se deducen de las relaciones entre sus miembros y los elementos críticos ya que en las percepciones individuales de los sujetos se basa el entendimiento de la realidad laboral; dichas percep- 
ciones, a su vez, influyen en la conducta y actitudes de los individuos de la organización. Aquí se incluyen los trabajos de Campbell (1970), Guion (1971) y Pritchard \& Karasick (1973), (Seisdedos, 1985).

Por último se encuentra el conjunto de percepciones globales (constructo personal y psicológico) que tiene el individuo de la organización y que es el reflejo de la interacción entre ambos. Lo importante es cómo percibe un sujeto su entorno, sin tener en cuenta cómo lo perciben otros; por tanto, es más una dimensión del individuo que de la organización (Seisdedos, 1985).

Otras definiciones más recientes, como la de Pérez de Maldonado (2006), plantean que el ambiente de trabajo o clima organizacional puede ser entendido como un fenómeno socialmente construido que surge de las interacciones individuo-grupo-condiciones de trabajo, dando como resultado un significado en las experiencias individual y grupal, ya que lo que pertenece y ocurre en la organización afecta e interactúa con todo. Los resultados organizacionales son precisamente consecuencia de estas interacciones que se dan de manera dinámica, cambiante y cargada de afectividad (Maldonado, 2006)

Desde las premisas anteriores, el clima organizacional, visto como un componente de productividad, es una realidad empresarial como la estructura, los niveles de jerarquía, el liderazgo, las normas, la cultura, entre otras, que caracterizan la vida de una organización y que cada colaborador entiende y describe a partir de sus propias percepciones; se podría decir entonces, que es una realidad organizacional perceptiva y cognitiva.
El clima resultante de esta realidad, induce a los colaboradores a tener determinados comportamientos respecto a la calidad de la labor ejercida y la aceptación sobre la razón de ser de la organización, que determina el sentido de pertenencia e impacta los indicadores de efectividad, eficiencia, eficacia, es decir el desempeño general de la organización. Por tal motivo una de las funciones más importantes de la gerencia, es la creación de un clima organizacional favorable, tanto físico como mental, que conduzca a los individuos a cooperar de forma voluntaria y puedan dar lo mejor de sí en el logro de los objetivos y metas planeadas por la organización.

La comprensión del fenómeno organizacional es un imperativo de todos los directivos que tienen responsabilidades en el manejo de recursos personales, encargados de generar un elemento integrador entre la organización y la sociedad moderna, donde el avance acelerado de la ciencia y la tecnología obliga a competir dentro de un mercado, cuya dinámica está marcada fundamentalmente por el desarrollo económico y social.

La gestión del talento humano ha tomado una gran importancia para las organizaciones, llevándolas a tomar conciencia y valorar la necesidad de establecer mejores condiciones laborales, y potenciar la calidad de vida de las personas que la integran. Variables como el clima organizacional, la satisfacción laboral y el desempeño, tienen especial relevancia pues contribuyen de forma transversal a la gestión del talento humano, para lo cual se necesitan líderes que contribuyan en la materialización del bienestar laboral y la potencialización del capital humano, que soporta la organización en concordancia con una sociedad moderna, 
donde los sistemas organizacionales marchan a la par de los procesos de transformaciones sociales, $y$, que constituyen un medio a través del cual la sociedad busca soluciones a los problemas que enfrenta.

Se puede afirmar que el clima organizacional es un elemento dinámico, que construyen los grupos en las organizaciones, y en el que se mantiene un equilibrio entre los aspectos objetivos (externos al sujeto) y los aspectos subjetivos (dimensión psicológica). Este proceso se describe y construye a partir de las percepciones que elaboran y trasmiten los sujetos al interactuar en el contexto laboral (Vega, et al. 2006).

\section{Cómo medir el clima organizacional}

Las organizaciones del siglo XXI, para lograr mantenerse y mejorar sus procesos productivos, deben capacitarse gradualmente con el fin de analizar el comportamiento de las mismas, más cuando hoy en día, se considera que las organizaciones son sistemas abiertos y socialmente complejos (Ávila, 2013), por lo tanto, la conducta de cada individuo debe crecer como un sistema social, incorporando los principios teóricos y metodológicos que permitan analizar críticamente los diferentes escenarios organizacionales de acuerdo con las necesidades de los mismas.

Para llevar a cabo el análisis de las diversas problemáticas sobre las dinámicas organizacionales, es necesario establecer procesos que se argumenten teórica y empíricamente con una metodología y estructura propia, que permitan abordar y explicar los fenómenos que se originan a partir de cada una de las actividades organizacionales como lo son el cambio, la adaptación y el aprendizaje, destacables en entornos flexibles.

Se conoce la existencia de numerosas investigaciones y autores, que históricamente se han interesado por construir modelos para la medición del clima organizacional, lo cual permite encontrar una gran variedad de clasificaciones que generan un consenso en torno a la idea de que todo modelo es una representación abstracta del conjunto de interacciones, que conceptual y metodológicamente se delimitan como objeto de conocimiento, pues los modelos vinculan una concepción estructural que permite representar la manera como se interrelacionan las variables determinantes con ciertos resultados (Delgado \& Vanegas, 2013).

Caldin (citado por Delgado \& Vanegas, 2013), dice que los modelos no cumplen con las características inherentes a la definición de un modelo en ciencia debido a que presentan problemas de incompletitud, es decir, son modelos no completos que contraen expresiones que no pueden ser ni verdaderas ni falsas.

Scheler (citado por Delgado \& Vanegas, 2013), dice que no cumplen con el consenso de sistematicidad propuesto por diferentes autores y que éstos se reformulan sin tener en cuenta aspectos conceptuales o metodológicos, que son propios e indispensables en cualquier modelo. Delgado \& Vanegas (2013) afirman que los modelos, en conclusión, surgen más de la experiencia de quienes han hecho de ellos una forma de trabajo, que de un análisis argumentado teóricamente. 
Las organizaciones necesitan intervenciones, originadas de estudios, que les permitan identificar las causas de los problemas diagnosticados en cada uno de los análisis que arrojen los estudios realizados. Esta gran demanda de intervención organizacional, se puede decir, ha permitido la creación de diferentes modelos de diagnóstico, entre ellos los "modelos de medición y diagnóstico de clima organizacional".

Se pueden referenciar algunos modelos como el modelo de comportamiento organizacional OBM, que se basa en la relación de contingencia establecida entre el comportamiento individual y el grupo. Este modelo incluye los siguientes supuestos teóricos:

a) Es imposible separar los supuestos teóricos del análisis organizacional del proceso de formulación; cada hipótesis representa una formulación derivada de enfoques teóricos e interpretación de los datos obtenidos de una fase experimental.

b) La coherencia teórica no es suficiente para demostrar la validez de un modelo; la única forma en la que un modelo puede demostrar su potencial predictivo es a través del éxito de la modificación de los indicadores de las problemáticas organizacionales y el mantenimiento de los efectos a través del tiempo.

c) Existe un principio de "parsimonia" cuando se elige un modelo de formulación a seguir ya que después de la fase exploratoria ningún modelo permite total certidumbre en sus efectos ni cubre todas y cada una de las variables implicadas en la problemática, pero permite jerarquizar las relaciones más importantes e intervenir sobre aquellas que poseen un espectro más amplio de efectividad.

d) El proceso de formulación debe ser coherente con el plan de intervención diseñados por el profesional, por eso, dicho plan tiene una gran carga de teoría innegable con técnicas de evaluación e interpretación de los datos según el enfoque teórico del análisis del comportamiento de la organización (Sandoval \& Cortés citado por Delgado \& Vanegas, 2013).

El modelo de los valores en competencias MVC, fundamentado en el modelo Quinn, fue utilizado en la investigación que realizó realizada por Hernández Sampieri, Méndez Valencia \& Contreras Soto (2014), y está basado principalmente en encontrar los principales indicadores de las organizaciones efectivas que pretenden resolver interrogantes como ¿Cuáles son los criterios para determinar si una organización es o no efectiva?, ¿Qué factores determinan la efectividad de una organización?, Cuando un individuo juzga una organización como efectiva, ¿qué indicadores tienen en mente? (Hernández Sampieri, Méndez Valencia \& Contreras Soto, 2014).

Elena Maish Molina (2004), propone que para resolver el primer interrogante, el modelo considera dos dimensiones: primero, flexibilidad, discreción y dinamismo versus estabilidad, orden y control; segundo: orientación interna, interacción y unidad versus orientación externa, diferenciación y rivalidad. Los cruces de estas dos dimensiones producen 
cuatro subdominios culturales, que dan origen al nombre del modelo porque los criterios que están dentro de cada cuadrante llevan mensajes contradictorios: los directivos quieren organizaciones adaptables y flexibles pero también que sean estables y controlables mientras que los subdominios, que parecen ser cuatro perspectivas completamente diferentes, están estrechamente relacionadas y entretejidas.

Por otro lado, los cuadrantes en el MVC representan los valores subyacentes que se encuentran por encima de los empleados, programas, políticas y organizaciones que existen. La mayoría de las empresas e instituciones tienen elementos diferentes pero desarrollan un estilo dominante. Por lo tanto, no se trata de un modelo tipológico sino topológico (Hernández Sampieri, Méndez Valencia \& Contreras Soto, 2014), mientras que Reinoso \& Araneda (2008) proponen un diseño para medición de clima organizacional que facilita dicho proceso, según los siguientes pasos:

Análisis de objetividad: que se obtiene a partir de la definición y estandarización de criterios para la aplicación y análisis de la información.

Análisis de la confiabilidad: se refiere al grado en el que el instrumento de medición produce resultados iguales, es decir, consistentes y coherentes. Se calcula a partir de coeficientes de confiabilidad que en general, oscilan entre cero y uno, donde 0 , representa inexistencia de confiabilidad y 1 el máximo de confiabilidad. En consecuencia, mientras más cercanos a 1 sean los coeficientes, mayor será la confiabilidad.
Análisis de la validez: este es el instrumento que mide el clima organizacional, y lo dividen en tres aspectos:

-Validez de contenido: es el grado en el cual la medición representa al concepto o variable medida. El dominio de contenido de una variable es definido por la literatura respecto al tema y se calcula con base en la correlación entre los ítems que miden la misma variable.

-Validez de criterio: establece la validez del instrumento de medición al compararlo con algún criterio externo que pretende medir lo mismo. Se estima correlacionando las mediciones del instrumento con las del criterio para la misma variable.

-Validez del constructo: se refiere a cuán exitosamente un instrumento representa y mide un concepto teórico. El instrumento debe explicar el modelo teórico empírico que subyace a la variable de interés. Esta validez le confiere el significado al instrumento, es decir, qué está midiendo y cómo está operando para medirlo. Se estima mediante análisis estadístico multivariado como análisis de factores, análisis discriminado, y otros (Reinoso \& Araneda, 2008).

\section{Impacto de la gestión humana en el clima organizacional}

Las organizaciones que buscan estar a la vanguardia requieren constantemente retroalimentación y evaluación sobre la base de sus 
juicios y equivocaciones, tener una capacidad de cambio y de reacción que amortigüe su dirección al entorno. Alcanzar excelentes indicadores en la gestión de una organización supone un interés sin límites por afinar la dirección que se ejerce acentuando el componente humano, en la disposición de los equipos y colectivos de trabajo y su interdependencia con las individualidades de cada quien, lo cual facilitará identidad y balance entre los objetivos e intereses de cada persona y de su colectivo, e incluso de la organización.

Las empresas que están expuestas a los constantes avances tecnológicos y a los cambios de las formas de trabajo están sujetas a premisas como la de Lambert (2001): “La excelencia de hoy es la mediocridad de mañana", lo cual toma hoy día un gran valor y sentido para las organizaciones.

Drucker por su parte, plantea: “Desde mediados de los años 70’s, lo que se conoce sobre administración ya no nos sirve [...] En el futuro inmediato, los gerentes tendrán que ser capaces de olvidar lo que hacían, tan rápido como aprenden cosas nuevas que tienen que hacer" (1985, p.6).

Más reciente Michael \& James, (1994), fundadores de la reingeniería, expresaron: "El problema de los negocios norteamericanos es que están entrando en el siglo XXI, con compañías diseñadas en el siglo XIX, para trabajar en el siglo XX".

Y hace sólo unos cuantos lustros Saravia (1996) dijo: "La velocidad de la innovación tecnológica lleva a la rápida obsolescencia del conocimiento. Los hallazgos científicos son rápidamente superados. Ello también ocurre con los conocimientos en materias de ciencias sociales y, específicamente en el campo de la administración. Nada más viejo que un texto de gerencia de hace diez años. El ciclo de vida de los modismos organizacionales es cada vez más breve" (pp.11-30).

\section{Conclusiones}

La medición del clima organizacional reconoce y determina fortalezas, debilidades y áreas de oportunidades que permiten establecer planes de acción para alcanzar los objetivos y metas organizacionales.

Dicho clima establece condiciones claras para lograr el desarrollo organizacional, ya que analiza aspectos básicos y esenciales de la empresa como la estructura, los puestos de trabajo, los canales y programas de motivación y de relaciones, siendo estas las dimensiones que se impactan con el nivel de desarrollo que se realicen en cada organización.

El clima organizacional es el resultado de varios factores, como la estructura organizacional, el liderazgo, la participación, las relaciones formales e informales, el comportamiento en el trabajo, entre otros, que son percibidos por los colaboradores dependiendo en la forma que les impacte en su vida laboral, personal, familiar y social.

La gestión del talento humano, con el tiempo y la evolución de las organizaciones, que las obliga a estar en constantes cambios, se ha convertido en un mecanismo de apoyo para la gerencia organizacional, que es trascendental para el manejo de las relaciones laborales, el 
fortalecimiento de la cultura organizacional y la generación de un buen clima organizacional, coherente con la filosofía y la identidad de la organización, que guíe una labor de calidad basada en la motivación de cada uno de sus integrantes, evidenciada en el sentido de pertenencia de los mismos, convirtiéndose así esta labor en uno de los indicadores más importantes de la gestión del talento humano.

\section{Referencias}

Ávila V, A. (2013). Las organizaciones como sistemas sociales complejos. Integración Académica en Psicología, 1(2). Recuperado de: https://integracion-academica.org/vol1numero2-2013/25-las-organizaciones-como-sistemas-sociales-complejos.

Cabrera, G. A. (1999). O clima organizacional nas empresas chilenas. Revista de Psicología Social e Institucional, 1(2). Recuperado de: http://www.uel.br/ccb/psicologia/revista/oclima.htm

Chiavenato, I. (2002). Gestión del talento humano. México: Mc Graw Hill.

Delgado, L. \& Vanegas, M. (2013). Psicología organizacional perspectivas y avances. Bogotá: Ecoe Ediciones.

Drucker, P. (1985). La gerencia. Tareas, responsabilidades y prácticas. Buenos Aires: El Ateneo.

Hernández Sampieri, R., Méndez Valencia, S. \& Contreras Soto, R. (2014). Construcción de un instrumento para medir el clima organizacional en función del modelo de los valores en competencia. Contaduría y Administración de la UNAM, 59(1), 229-257.

Lambert, T. (2001). Manual de consultoría: cómo iniciarse y crecer en el mundo de la consultoría. Barcelona: Gestión.

Maish Molina, E. (2004). Pautas Metodológicas para la realización de Estudios de Clima Organizacional. Gestión en el Tercer Milenio, 13(7), 35-38.

Michael, H. \& James, C. (1994). Reingeniería. Bogotá: Grupo Editorial Norma.

Mladinic, A. I. (2011). Justicia organizacional: entendiendo la equidad en las organizaciones. Psykhe, 11(2). Recuperado de: http:// www.psykhe.cl/index.php/psykhe/article/view/428/407

Naranjo, J. V. (2014). Cultura organizacional e innovación: estudio empírico de la ONU. Recuperado de: http://www.researchgate.net/ publication/28231970_Cultura_organizacional_e_innovacin_un_estudio_emprico

Patlán-Pérez, J., Martínez, E. \& Hernández, R. (2012). El clima y la justicia organizacional y su efecto en la satisfacción laboral. Revista Internacional Administración \& Finanzas, 5(5), 1-19.

Pérez de Maldonado, I. a. (2006). Clima organizacional y gerencia: inductores del cambio organizacional. Investigación y Postgrado, 21(2), 231-248. 
Reinoso, H. \& Araneda, B. (2008). Diseño y validación de un modelo de medición del clima organizacional basado en percepciones y expectativas. Revista Ingeniería Industrial, $1,-39-54$.

Robbins, S. (2004). Comportamiento organizacional. México: Pearson.

Saravia, E. (1996). Las escuelas e institutos de administración pública en América Latina frente a la crisis del Estado. Situación actual y perspectivas de transformación. REGAP: Revista galega de administración pública, 13, 11-30.

Seisdedos, N. (1985). El clima laboral y su medida. Madrid: Colegio Oficial de Psicólogos de Madrid.

Vega D., Arévalo. A., Sandoval, J., Aguilar, M. C. \& Giraldo, J. (2006). Panorama sobre los estudios de clima organizacional en Bogotá (1994-2005). Diversitas. Perspectivas en Psicología, 2(2), 329-349.

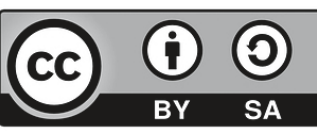

